Check for updates

Cite this: RSC Adv., 2017, 7, 53871

\title{
Single-faced flame resistance of cotton fabrics modified via mist copolymerization
}

\author{
Zewen Yang, Yanyan Zhang, Feiya Fu and Xiangdong Liu (DD *
}

Cotton fabrics with single-faced flame resistance are successfully fabricated through a simple mist copolymerization process using pentabromobenzyl acrylate (PBBA) as the functional monomer. The comonomers are methyl acrylate (MA), which can react with the hydroxyl groups of cellulose by transesterification, and divinyl benzene (DVB), a cross-linker. SEM images indicate that a very thin copolymer layer (the thickness is about $200 \mathrm{~nm}$ ) was formed on the cotton fiber surface and the flame resistance tests show that the modified fabrics have an improved flammability with longer time to ignition (TTI), lower peak heat release rate (PHRR), lower total heat release (THR), and lower average mass loss rate (AMLR), when compared to the original cotton fabric. The modification also results in good wearing durability because the flame-retardant coating was covalently linked to the cotton fabric surface by many ester groups. Moreover, desired cotton characteristics such as tensile strength, water absorbency, vapor permeability and flexibility are mostly retained because the mist method gives a single-faced modification of the cotton fabrics.

Received 17th October 2017 Accepted 16th November 2017

DOI: 10.1039/c7ra11461c

rsc.li/rsc-advances process, and cause serious environmental problems; (4) the contact of the coatings with skin often induces toxic side effects, and (5) the industrial scales are hard to achieve in low cost.

In our previous works, ${ }^{43,44}$ a "mist polymerization" technique has been used to modify cotton fabric surface. Asymmetrically superhydrophobic, ${ }^{45,46}$ and wear-resistant ${ }^{47}$ cotton fabrics were fabricated by feeding atomized monomer solutions to an in situ polymerization to build thin polymeric coatings on the cotton fabrics. The advantages of the mist polymerization include wider range of applicable monomers, tailorable coating thinness and surface morphology, simple operation, single faced modification, and almost no damage to the original properties of the fabric. These features of the mist polymerization method are suitable for fabricating cotton fabric with single faced function.

Pentabromobenzyl acrylate (PBBA) is a polymerizable monomer containing of approximately 71 wt $\%$ bromine element, ${ }^{48-50}$ belonging to the class of organobromine FR, which appears to work even at low concentration. ${ }^{50}$ Both homopolymerization $^{50-52}$ and copolymerization ${ }^{53,54}$ of PBBA were widely studied for the applications in commercial polymer materials such as polystyrene, polyamide 6 , and polypropylene.

In this work, a mist copolymerization process utilizing PBBA as the FR monomer is applied to modify cotton fabrics. Methyl acrylate (MA) is used as a co-monomer to enhance the adhesion of the polymer coatings via a transesterification with the hydroxyl groups of cellulose. The flame resistance properties of the resulting cotton fabrics are examined by the burning tests and cone calorimeter experiments. The abrasion resistance, mechanical stability, water absorbability, and moisture transmissibility of the modified cotton fabrics are further characterized. 


\section{Experimental}

Materials

Pentabromotoluene (PBB) was obtained from TCI Co., Ltd (Shanghai, China). The cotton fabrics were purchased from a local fabric store (60 ends per $\mathrm{cm}, 30$ picks per $\mathrm{cm}, 0.42 \mathrm{~mm}$ thickness, $120 \mathrm{~g} \mathrm{~m}^{-2}$ weight, $35.2 \mathrm{~m}^{2} \mathrm{~g}^{-1}$ specific surface area). Before chemical modification, the cotton samples were cleaned by ultrasonic washing in ethanol (30 $\mathrm{min}$ ) and deionized water (30 min $\times 3$ times), respectively. Other chemical reagents were purchased from Aladdin Co., Ltd (Shanghai, China), and all used as received without further purification. Deionized water with a resistivity of $18.2 \mathrm{M} \Omega \mathrm{cm}$ was used in all experiments.

\section{Synthesis of pentabromobenzyl bromide (PBBBr)}

PBB (19.6 g, $40.3 \mathrm{mmol}$ ), $N$-bromosuccinimide (NBS, $7.0 \mathrm{~g}, 39.3$ $\mathrm{mmol})$, liquid bromine $\left(\mathrm{Br}_{2}, 1.0 \mathrm{~mL}\right)$, benzoyl peroxide (BPO, $2.0 \mathrm{~g}, 8.3 \mathrm{mmol})$, and carbon tetrachloride $\left(\mathrm{CCl}_{4}, 100 \mathrm{~mL}\right)$ were mixed in a three-neck glass flask $(250 \mathrm{~mL})$ in an oil bath, heated at $80{ }^{\circ} \mathrm{C}$ for $1 \mathrm{~h}$, added with aqueous hydrogen peroxide $30 \%$ $\left(\mathrm{H}_{2} \mathrm{O}_{2}, 11 \mathrm{~mL}\right)$, and stirred for $4 \mathrm{~h}$. The produced precipitate was washed with $\mathrm{CCl}_{4}(80 \mathrm{~mL} \times 3$ times $)$, and dried in a vacuum oven at $80{ }^{\circ} \mathrm{C}$ for $12 \mathrm{~h}$ to obtain the product. Yield $=86.4 \% ;{ }^{1} \mathrm{H}$ NMR (400 MHz, $\left.\mathrm{CDCl}_{3}\right)$ : 5.01 (-CH$\left.{ }_{2} \mathrm{Br}\right)$; FTIR (KBr): 2957, 1698, $1429,642 \mathrm{~cm}^{-1}$.

\section{Synthesis of pentabromobenzyl acrylate (PBBA)}

PBBBr (21.2 g, $37.5 \mathrm{mmol})$, a mixture solution of acrylic acid (AA) and sodium acrylate (NaAC) $\left(10.4 \mathrm{mmol} \mathrm{L}^{-1}, \mathrm{pH}=6.5\right.$, $4.6 \mathrm{~mL}$ ), and 2-methoxyethanol (ME, $63 \mathrm{~mL}$ ) were mixed, heated at $100{ }^{\circ} \mathrm{C}$ for $3 \mathrm{~h}$. The obtained precipitate was washed with deionized water $(100 \mathrm{~mL} \times 3$ times $)$, and dried in a vacuum oven at $80{ }^{\circ} \mathrm{C}$ for $12 \mathrm{~h}$ to obtain the product. Yield $=93.5 \% ;{ }^{1} \mathrm{H}$ NMR (400 MHz, $\left.\mathrm{CDCl}_{3}\right)$ : 6.45 and $5.90\left(=\mathrm{CH}_{2}-\right), 6.15(=\mathrm{CH}-)$, 5.69 (- $\left.\mathrm{CH}_{2} \mathrm{O}-\right)$; FTIR (KBr): 3031, 2900, 1730, 1465, $985 \mathrm{~cm}^{-1}$.

\section{Typical procedure of mist polymerization on the cotton surface}

PBBA (0.2 g, $0.36 \mathrm{mmol}$ ), methyl acrylate (MA, $31 \mathrm{mg}$, $0.36 \mathrm{mmol}$ ) and divinylbenzene (DVB, $7.2 \mu \mathrm{mol})$ were dissolved in acetone $(15 \mathrm{~mL})$, and used as the monomer solution. An aqueous solution of ammonium ceric nitrate (ACN) $(3.0 \mathrm{~mL}$,
18.2 $\mathrm{mmol} \mathrm{L}^{-1}$ ) was atomized using an air compression-type atomizer (DH-M01, DongHan, China), fed $\left(0.36 \mathrm{~mL} \mathrm{~min}^{-1}\right)$ to a side surface of a cotton fabric sample $(30 \times 30 \mathrm{~mm})$ for $5 \mathrm{~min}$, and dried at $80{ }^{\circ} \mathrm{C}$ for $10 \mathrm{~min}$ to obtain ACN treated cotton fabric. The monomer solution was atomized, fed $(0.8$ $\mathrm{mL} \mathrm{min}^{-1}$ ) to the ACN cotton sample for $5 \mathrm{~min}$, heated at $60{ }^{\circ} \mathrm{C}$ overnight and at $180{ }^{\circ} \mathrm{C}$ for $5 \mathrm{~min}$, washed with deionized water $\left(50 \mathrm{~mL} \times 3\right.$ times), dried at $80{ }^{\circ} \mathrm{C}$ for $2 \mathrm{~h}$. Other modified cotton fabric samples were prepared using the procedures shown in Table 1. Fully modified cotton fabric was prepared under a similar process but changed the mist feeding step with an immersion treatment in the monomer solution.

\section{Characterizations}

Fourier transform infrared (FTIR) measurements were performed on a Nicolet Avatar 370 spectrometer (Nicolet Company, Madison, USA) in a normal transmission mode. ${ }^{1} \mathrm{H}$ NMR spectra were recorded on an Avance AV-400 (400 MHz) NMR spectrometer (Bruker, Switzerland) in $\mathrm{CDCl}_{3}$ with TMS as an internal standard. Fabric surface was observed by a JSM6700F field emission scanning electron microscope (FE-SEM, JEOL, Japan) after gold coating (thickness of approximately $10 \mathrm{~nm}$ ). Attenuated total reflectance infrared (ATR-IR) spectra were collected utilizing a Nicolet Avatar 370 spectrometer (Nicolet Company, Madison, USA) equipped with an ATR accessory. Fabric abrasion tests were performed using a Martindale abrasion instrument (HZ-8029A, Heng Zhun Instrument Co., Ltd, China) according to the method of DIN EN ISO 12947-3.

The vertical burning test was carried out directly burning the fabric for $10 \mathrm{~s}$ in the flame of alcohol burner, referring to the standard test method ENISO 15025. The cotton fabric samples was folded to make the modified surface outward to flame (before burning), and the burning state of the fabrics was recorded by a camera. To compare the flammability of the cotton samples with and without FR modification, a cotton fabric $(60 \times 60 \mathrm{~mm})$ having half-modified surface was prepared by combining the mist polymerization process (same to FRcotton4) with a shielding over the other half to keep the original cotton surface. To check the durability of the FR coating, the modified fabric samples after 150 abrasion cycles (abrasion conditions were same to previous works ${ }^{\mathbf{4 0 - 4 2}}$ ) were evaluated using the flame resistance test described above.

Table 1 Preparation conditions and burning rate of the fabric samples

\begin{tabular}{|c|c|c|c|c|}
\hline Sample & Finishing methods & Monomers & Burning rate $\left(\mathrm{mm} \mathrm{s}^{-1}\right)$ & Burning rate ${ }^{b}\left(\mathrm{~mm} \mathrm{~s}^{-1}\right)$ \\
\hline Pristine cotton & - & - & $3.00 \pm 0.03$ & $3.00 \pm 0.03$ \\
\hline FR-cotton 1 & Mist $^{a}$ & PBBA, DVB & $1.76 \pm 0.08$ & $3.00 \pm 0.09$ \\
\hline FR-cotton 2 & Mist & PBBA, DVB, AA (24 mmol L $\left.\mathrm{m}^{-1}\right)$ & $1.70 \pm 0.07$ & $2.24 \pm 0.11$ \\
\hline FR-cotton 3 & Mist & PBBA, DVB, MA $\left(12 \mathrm{mmol} \mathrm{L}^{-1}\right)$ & $1.66 \pm 0.08$ & $1.98 \pm 0.11$ \\
\hline FR-cotton 4 & Mist & PBBA, DVB, MA $\left(24 \mathrm{mmol} \mathrm{L}^{-1}\right)$ & $1.60 \pm 0.07$ & $1.72 \pm 0.08$ \\
\hline FR-cotton 5 & Mist & PBBA, DVB, MA $\left(36 \mathrm{mmol} \mathrm{L}^{-1}\right)$ & $1.65 \pm 0.08$ & $1.72 \pm 0.09$ \\
\hline FR-cotton 6 & Immersion $^{c}$ & PBBA, DVB, MA $\left(24 \mathrm{mmol} \mathrm{L}^{-1}\right)$ & $1.57 \pm 0.06$ & $1.76 \pm 0.11$ \\
\hline
\end{tabular}

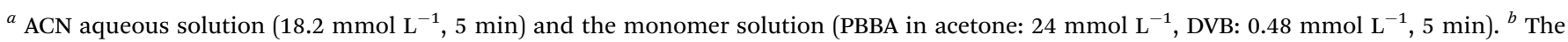
burning rate of the sample after 150 abrasion cycles. ${ }^{c}$ ACN treated for 15 min and the monomer solution treated for 30 min. 
In order to further verify the flammability, the combustion behavior of the cotton fabrics was also evaluated by cone calorimeter (FTT Company, UK) with a heat flux of $35 \mathrm{~kW} \mathrm{~m}^{-2}$. The flammability indexes of total heat release (THR), peak of heat release (PHRR), time to ignition (TTI), and average mass loss rate (AMLR) were simultaneously measured. According to the ISO 5660 standard, the samples were cut into the size of $100 \times$ $100 \mathrm{~mm}^{2}$ and wrapped with aluminum foil and placed in a frame with grid. The measurement was repeated to triplicate and the average data were reported.

Water absorption ability, water vapor permeability, tensile strength, and flexibility of the modified cotton fabrics were determined using the methods reported in our pervious works. ${ }^{\text {43-47 }}$

\section{Results and discussion}

\section{Synthesis of the flame retardant monomer}

As shown in Fig. 1a, the PBBA monomer was synthesized by reacting $\mathrm{AA}$ with $\mathrm{PBBBr}$, which was obtained by brominating PBB. Fig. 1b shows the FTIR spectra of PBBA and PBBBr. The peaks at $624 \mathrm{~cm}^{-1}$ and $1429 \mathrm{~cm}^{-1}$ in the spectrum of $\mathrm{PBBBr}$ are attributable to the $\mathrm{CH}_{2}-\mathrm{Br}$ stretching and $-\mathrm{CH}_{2}$ bending, respectively. However, these peaks disappeared in the spectrum of PBBA, and were displaced by a strong absorption peak at $1730 \mathrm{~cm}^{-1}$, meaning that the $\mathrm{CH}_{2}-\mathrm{Br}$ structure has been substituted by an acrylate group. The molecular structure of PBBA was further confirmed by the ${ }^{1} \mathrm{H}-\mathrm{NMR}$ spectra shown in Fig. 1c. The peak at $5.01 \mathrm{ppm}$ is attributable to the methylene groups in $\mathrm{PBBBr}$, and the peak at $5.69 \mathrm{ppm}$ is corresponded to the $-\mathrm{CH}_{2} \mathrm{CO}_{2}-$ structure in PBBA. In addition, the peaks at
$5.90 \mathrm{ppm}$ and $6.45 \mathrm{ppm}$ are believed due to the cis and trans structures of $=\mathrm{CH}_{2}$, and the peak at $6.15 \mathrm{ppm}$ is assignable to the proton in the $=\mathrm{CH}$ - bond.

\section{Fabrication of the FR cotton surfaces}

The FR layer on the cotton fabric was obtained through a twostep procedure (Scheme 1). The oxidation of cellulose by ACN gives radicals first. Then the mist copolymerization, which was initiated by the resulting radicals, forms the copolymer layer on the cotton fiber surface. The three monomers in the mist copolymerization play respective roles: DVB is used as a crosslinker; MA is designed to introduce ester groups to react with the hydroxyl groups of cellulose; and PBBA acts as the functional monomer to decrease the flammability of cotton fabric.

Our previous works ${ }^{45,46}$ reported that the diameters of the mist droplets range from 150 to $500 \mathrm{~nm}$, and only a small number of the droplets (about 3\%) is fixed on the cotton surface during the mist feeding. Therefore, mist copolymerization generally results a thin polymeric layer on a single side surface of the substrate.

Five fabric samples (Table 1) were prepared using mist polymerization, and their modified surfaces were examined using ATR-IR surface analysis technique. Fig. 2 compares the ATR-IR spectra with a comparison with pristine cotton. One characteristic peak appeared at $1725 \mathrm{~cm}^{-1}$ in the spectra of modified cotton fabrics but not in the spectra of pristine cotton fabric and the opposite surfaces (data not shown). This peak is attributable to the covalent bond of $\mathrm{C}=\mathrm{O}$ in the ester structure, meaning that the mist copolymerization took place on the single side surface of the cotton fabric.

a

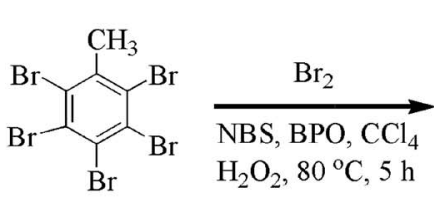

PBB<smiles>BrCc1c(Br)c(Br)c(Br)c(Br)c1Br</smiles>

$\mathrm{PBBBr}$

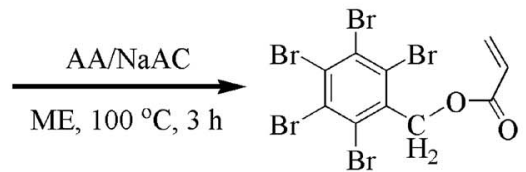

PBBA
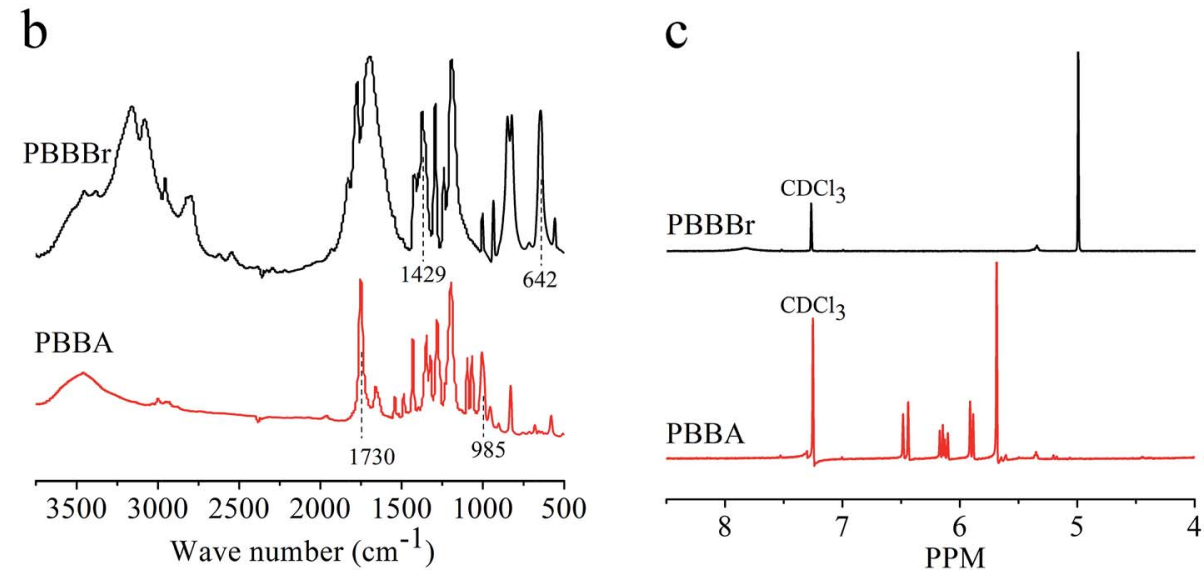

Fig. 1 The synthesis of PBBA monomer (a), FTIR spectra of PBBBr and PBBA (b), ${ }^{1} \mathrm{H}$ NMR spectra of PBBBr and PBBA (c). 


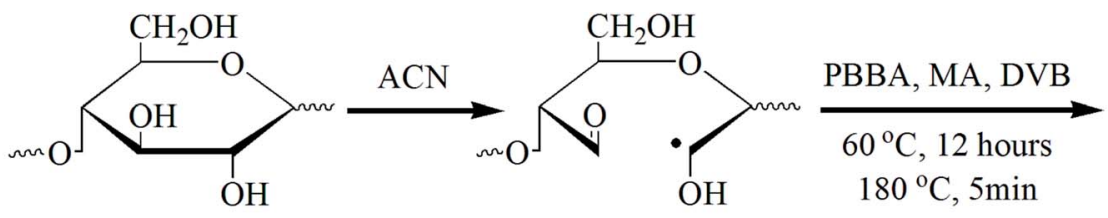
Cellulose<smiles>C=CC(=O)OCc1c(Br)c(Br)c(Br)c(Br)c1COC(=O)C=C</smiles>

Scheme 1 Scheme of the surface modification on a single side of cotton fabric.

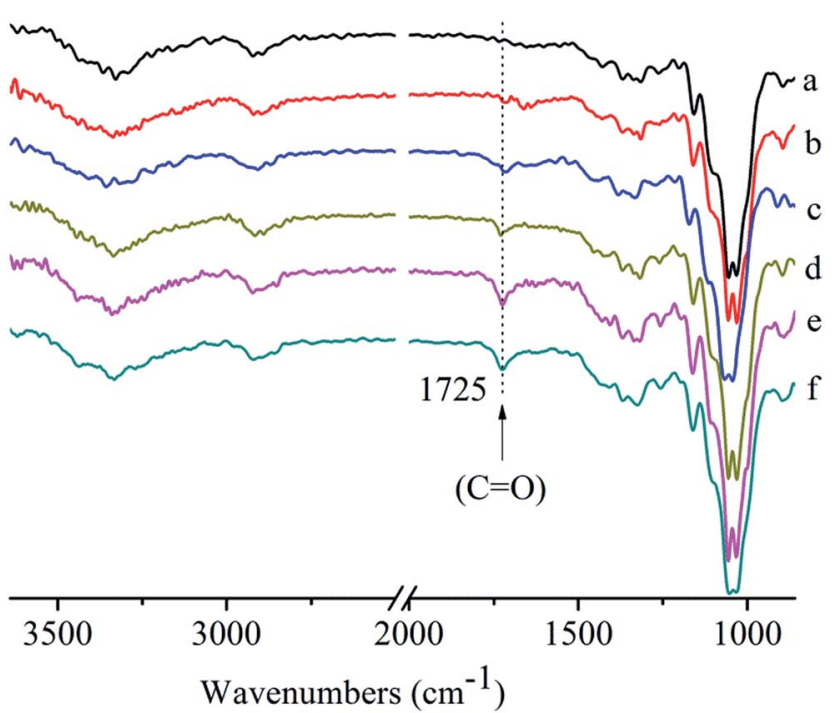

Fig. 2 ATR-IR spectra of pristine cotton (a), FR-cotton1 (b), FRcotton2 (c), FR-cotton3 (d), FR-cotton4 (e), FR-cotton5 (f).

The low-magnification SEM images for pristine cotton and FR-cotton4 (Fig. 3a and b) display nothing significantly different, meaning that the copolymer layer formed on the cotton fiber surface was very thin. The high-magnification SEM images (Fig. 3c-f) further suggest that the copolymer layer possesses a thinness of approximately $200 \mathrm{~nm}$.

\section{Flame resistance of the FR cotton surfaces}

First, the flame resistance of the modified surfaces was tested by burning the fabrics in a flame for $10 \mathrm{~s}$ (Fig. 4) as an improved method basing on the standard test method ENISO 15025. Before the burning test, the fabric sample was folded to make the modified surface outward to flame, and the whole burning process was recorded by an optical camera. As a result, the pristine cotton fabric (Fig. 4a) was almost completely burned in $10 \mathrm{~s}$, whereas the modified cottons (Fig. 4b-d) were partly burned. As shown in Table 1, in contrast with the pristine cotton (burning rate was $3 \mathrm{~mm} \mathrm{~s}^{-1}$ ), all the modified fabric samples exhibited significant flame resistance effect, especially, FRcotton 4 slowed the burning rate to $1.60 \mathrm{~mm} \mathrm{~s}^{-1}$. To investigate wearing durability of the FR coatings, the modified

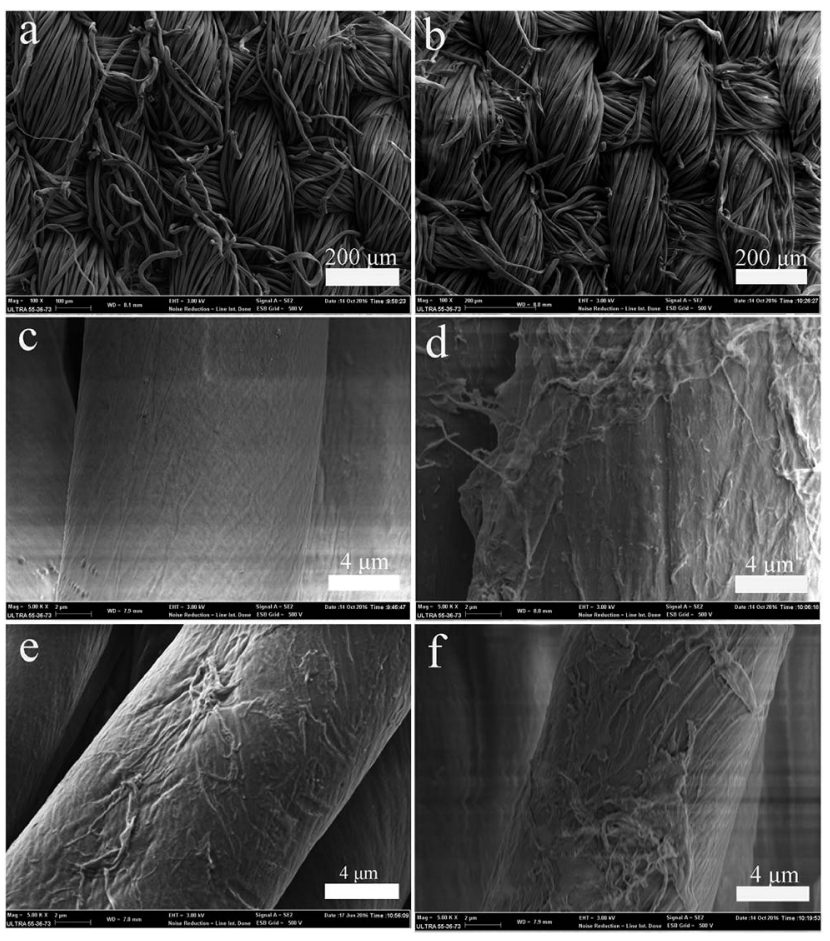

Fig. 3 Low magnification SEM images of pristine cotton (a) and FRcotton4 (b); high magnification SEM images of pristine cotton (c), FRcotton1 (d), FR-cotton2 (e), and FR-cotton4 (f). 


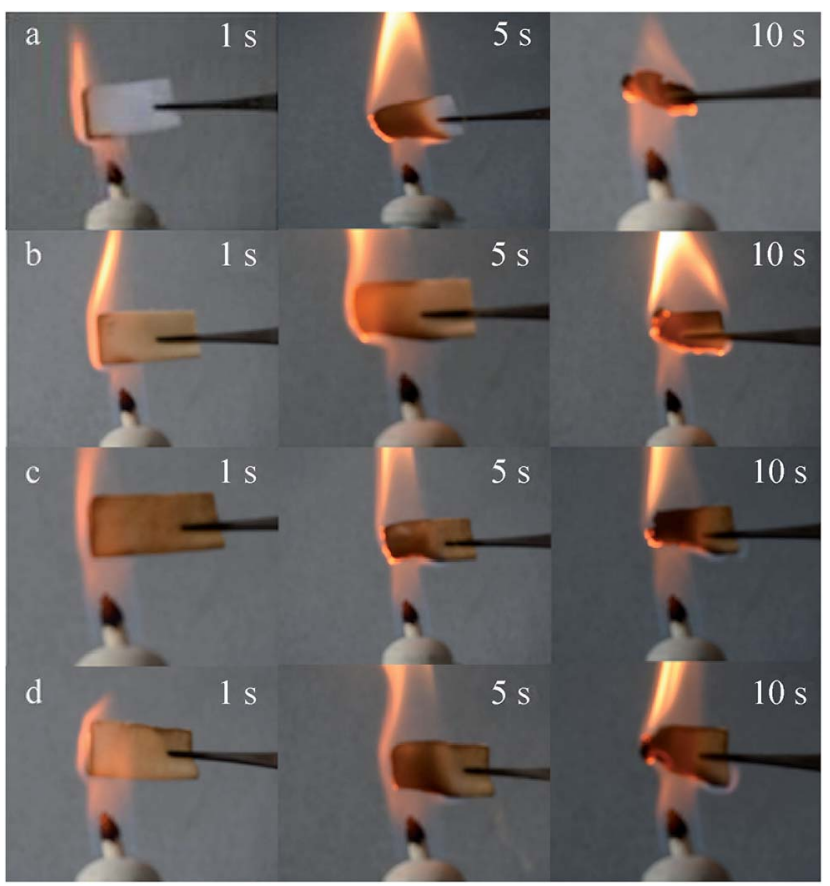

Fig. 4 Photographs of the cotton fabrics in burning. (a) Pristine cotton, (b) FR-cotton1, (c) FR-cotton2, and (d) FR-cotton4.

surfaces were abraded using a Martindale abrasion instrument. The flame resistance effect of the samples after 150 abrasion cycles was evaluated again using the improved flame resistance method. As shown in Fig. 5, FR-cotton1 (Fig. 5b) was burning fast as pristine cotton (Fig. 5a), suggesting that the FR function was lost after the repeated abrasion tests. In contrast, the

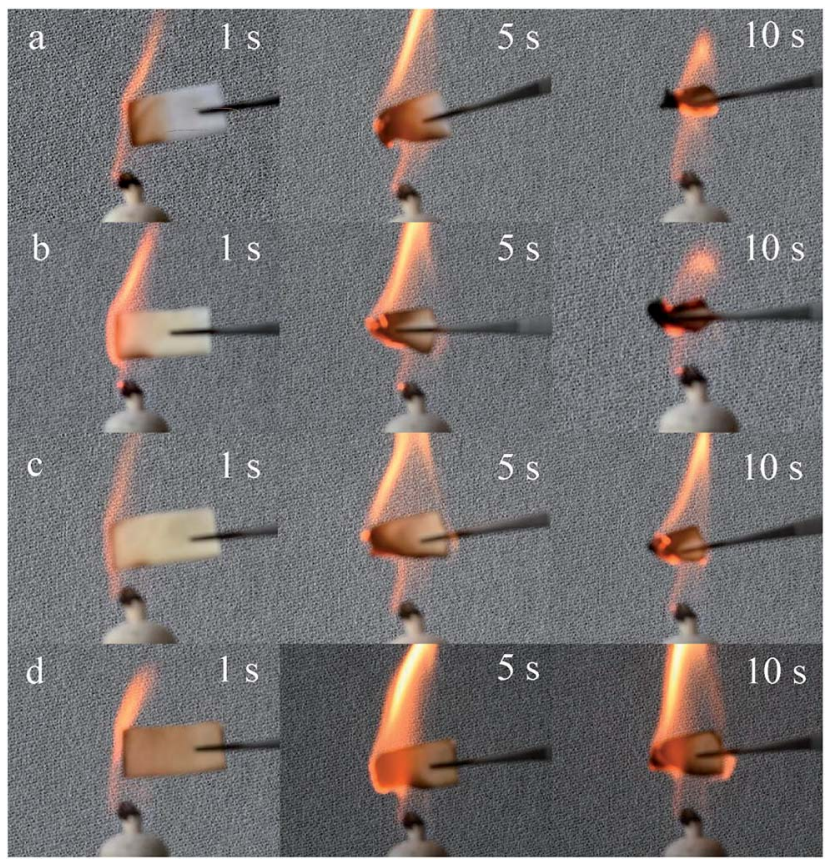

Fig. 5 Photographs of the cotton fabrics (after the abrasion test) in burning. (a) Pristine cotton, (b) FR-cotton1, (c) FR-cotton2, and (d) FRcotton4. copolymer coating containing MA monomer showed a promising abrasion resistance (Fig. 5d). The reason may be assigned to the covalent ester linkages between the copolymer layer and the cellulose chains. When compared with FR-cotton3 (Table 1), FR-cotton4 exhibited more satisfactory stability against repeated abrasion tests. In spite of the increased MA concentration, FR-cotton5 showed the durability similar to FR-cotton4, meaning that $24 \mathrm{mmol} \mathrm{L}^{-1}$ of the MA concentration was enough to enhance the durability against abrasion. After 150 cycles of the abrasion tests under a pressure of $12 \mathrm{kPa}$, the fabric sample still has good flame retardance (losing $7.5 \%$ in burning rate). This result suggested that the modified fabric can be used for practical applications such as curtain. ${ }^{4,55}$ Moreover, the addition of monomer AA (FR-cotton2) imparted the FR coating without satisfying improvement on the wearing durability (Fig. 5c), suggesting that the transesterification of the MA units occurred more easily than the esterification of the AA units. To further compare the flammability of the modified surfaces, a cotton fabric $(60 \times 60 \mathrm{~mm})$ with a shelter over its half area, was subjected to the mist copolymerization process (condition is same to FR-cotton4) to make the FR layer coated on the other area of the fabric surface. This special fabric was folded to align the two areas, suspended above the flame to fire it, and quenched immediately after it was ignited for 1.0 seconds. Its burned hollow shown in Fig. 6a indicates that the modified area has improved FR effect by comparing with the original part, suggesting that the copolymer layer on cotton surface can prolong the ignition time. On the other hand, the burning result shown in Fig. $6 \mathrm{~b}$ indicates that the opposite surface of the fabric has a burning rate same to original cotton. Because the mist copolymerization gives a single-side surface modification, this result is very understandable.

To estimate the combustion properties using cone calorimetry method, cotton fabric samples were wrapped with aluminum foil to make the modified surface up on a specimen holder. Fig. 7 shows the heat release rate (HRR) curves of pristine cotton and FR-cotton 4 at a heating flow of $35 \mathrm{~kW} \mathrm{~m}^{-2}$, and the cone calorimetry data were collected in Table 2. Cotton fabric is a flammable material, its peak heat release rate (PHRR)

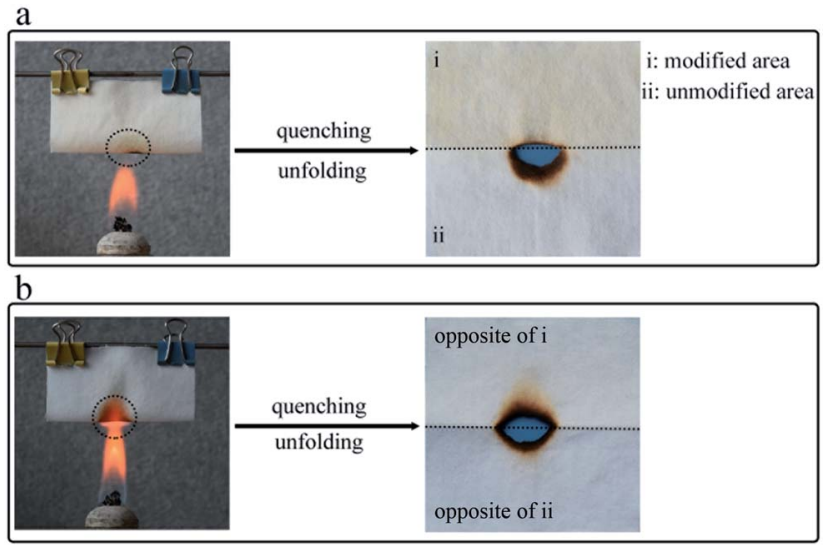

Fig. 6 Photographs of the combustion tests of the special cotton fabric. (a) The modified area was outward to flame. (b) The modified area was inward to flame. 


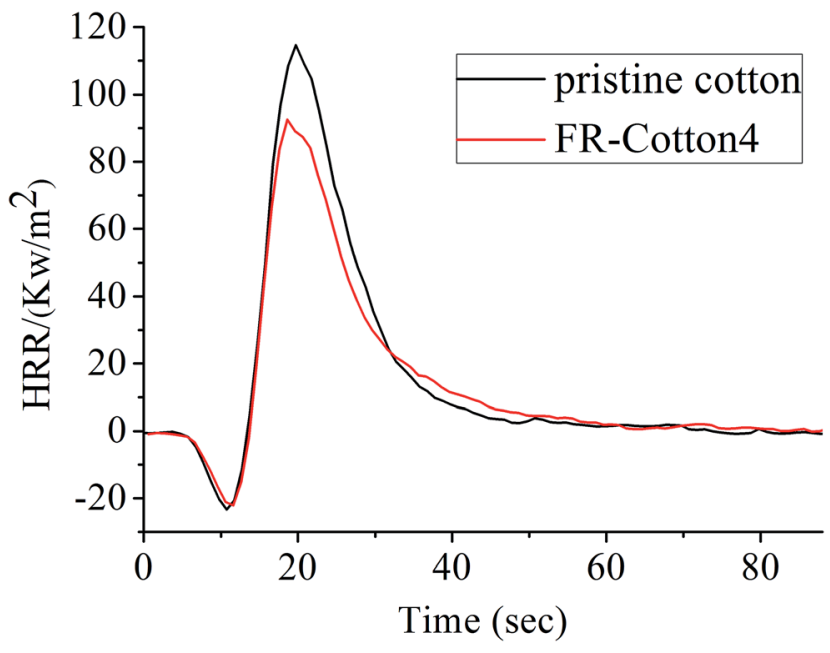

Fig. 7 HRR curves for the pristine cotton and FR-cotton4.

reaches a value $\approx 114 \mathrm{~kW} \mathrm{~m}^{-2}$. In comparison, the PHRR of the FR-cotton 4 was reduced by about $23 \%$. The time to ignition (TTI) of the FR-cotton 4 is $6 \mathrm{~s}$, longer than that of the pristine cotton. Both the total heat release (THR) and the average mass loss rate (AMLR) were slightly reduced, indicating again that the copolymer layer has an effective FR function for the cotton textile.

\section{Influences on the intrinsic properties of cotton}

As shown in Fig. 8i, the pristine cotton samples are of excellent water absorptivity (about 260\%), but the samples prepared using immersion method (FR-cotton6) are poor at $130 \%$. In contrast, the cotton fabrics modified by the mist copolymerization show good water absorptivity ranging from 245 to $218 \%$, which is slightly lower than that of the original cotton fabric. These results suggest that the single sided modification keeps a large part of the excellent water absorptivity of cotton. For most clothing products, the desired water absorption can lower wetting of sweat, thereby being pleasant for the wearer.

The vapor transmission rate of pristine cotton fabrics is $1450 \pm 48 \mathrm{~g}$ per $\mathrm{m}^{2}$ per day (Fig. 8ii), indicating that the original cotton fabric has good permeability to water vapor. However, the vapor transmission rate was decreased to about $58 \%$ by the immersion treatment (FR-cotton6, $850 \pm 43 \mathrm{~g}$ per $\mathrm{m}^{2}$ per day). Comparatively, the mist copolymerization process gave acceptable vapor transmission rates above $1300 \mathrm{~g}$ per $\mathrm{m}^{2}$ per day, which is very near to that of the original cotton fabric $(90 \%)$.

Table 2 Cone calorimetry data of the fabric samples ${ }^{a}$

\begin{tabular}{lllll}
\hline Sample & $\begin{array}{l}\text { TTI } \\
(\mathrm{s})\end{array}$ & \multicolumn{1}{l}{$\begin{array}{l}\text { PHRR } \\
\left(\mathrm{kW} \mathrm{m}^{-2}\right)\end{array}$} & $\begin{array}{l}\text { THR } \\
\left(\mathrm{MJ} \mathrm{m}^{-2}\right)\end{array}$ & $\begin{array}{l}\text { AMLR } \\
\left(\mathrm{g} \mathrm{s}^{-1}\right)\end{array}$ \\
\hline Pristine cotton & 4 & $113.97 \pm 6$ & $13.46 \pm 0.7$ & $0.024 \pm 0.003$ \\
FR-cotton4 & 6 & $92.51 \pm 5$ & $12.42 \pm 0.5$ & $0.020 \pm 0.003$
\end{tabular}

${ }^{a}$ TTI: time to ignition; PHRR: peak heat release rate; THR: total heat release; AMLR: average mass loss rate.
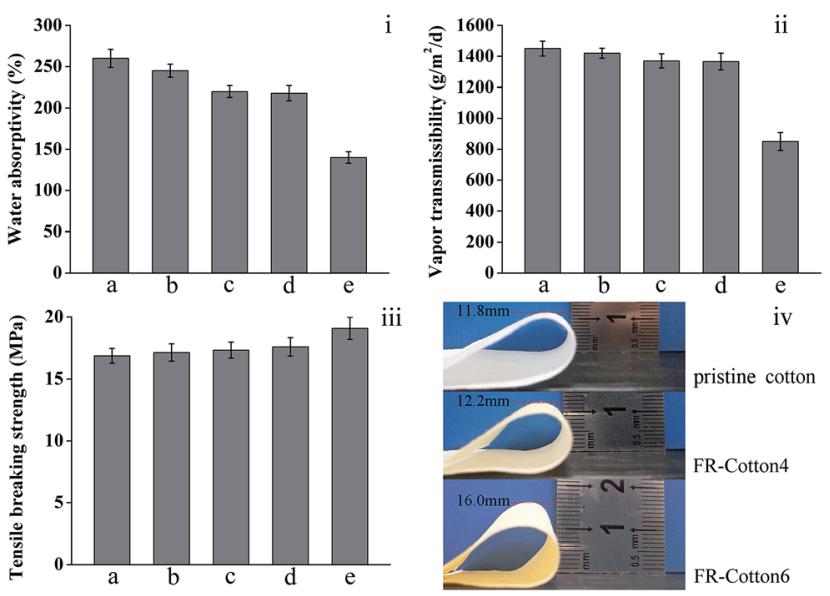

Fig. 8 The comparison in water absorbability (i), water vapor transmissibility (ii), tensile strength (iii), and flexibility (iv) of the cotton fabrics. (a) Pristine cotton, (b) FR-cotton1, (c) FR-cotton2, (d) FRcotton4, and (e) FR-cotton6.

The mechanical properties of the cotton fabrics were also studied by measuring the breaking tensile strength. As shown in Fig. 8iii, the pristine cotton fabric has a general breaking strength of $16.85 \mathrm{MPa}$, whereas the FR-cotton4 was lightly strengthened to $17.31 \mathrm{MPa}$, indicating that the mechanical damages caused by the finishing treatments are quite small.

Fig. 8iv compares the flexibilities of the modified cotton fabrics. The original cotton fabric exhibited a good flexibility, as the height of the loop less than $11.8 \mathrm{~mm}$. While the FR-cotton6 revealed a large loop height of more than $16.0 \mathrm{~mm}$, meaning that the flexibility damage caused by the immersion method is serious. The sample obtained by the mist copolymerization process (FR-cotton4) has a loop height of $12.2 \mathrm{~mm}$, which is very near to that of the original cotton fabric, suggesting that the mist copolymerization impaired the cotton flexibility insignificantly.

\section{Conclusions}

A new type of FR cotton fabric with single faced function was fabricated through a mist copolymerization technique. Unlike most other flame-retardant fabrics, this fabric exhibits asymmetric FR property on two faces: one face is of FR function but the opposite same to original cotton. The modified cotton fabrics delay the burning rate in the combustion tests and show positive FR behavior (including TTI, PHRR, THR and AMLR) in the cone calorimeter experiments. Moreover, the single face modification gives not serious damages on the desired cotton natures such as water absorption and vapor transmissibility. Considering the excellent balance of the new FR function and the intrinsic cotton natures, the method demonstrated in this work is believed to have promising potential in textile industries.

\section{Conflicts of interest}

There are no conflicts of interest to declare. 


\section{Acknowledgements}

This work was financially supported by the Natural Science Foundation of China (51573167) and Public Welfare Technology Application Research Project of Zhejiang Province (2017C31035 and 2017C33154).

\section{References}

1 Y. Y. Zhang, Q. B. Xu, F. Y. Fu and X. D. Liu, Cellulose, 2016, 23, 2791-2808.

2 Y. Gao and R. Cranston, Text. Res. J., 2008, 78, 60-72.

3 Y. L. Lam, C. W. Kan and C. W. M. Yuen, Text. Prog., 2012, 44, 175-249.

4 Q. B. Xu, L. J. Xie, H. L. Diao, F. Li, Y. Y. Zhang, F. Y. Fu and X. D. Liu, Carbohydr. Polym., 2017, 177, 187-193.

5 I. Ahmad and C. W. Kan, Materials, 2016, 9, 892.

6 A. Milionis, E. Loth and I. S. Bayer, Adv. Colloid Interface Sci., 2016, 229, 57-79.

7 Z. Xue, M. Liu and L. Jiang, J. Polym. Sci., Part B: Polym. Phys., 2012, 50, 1209-1224.

8 M. C. Wu, B. H. Ma, T. Z. Pan, S. S. Chen and J. Q. Sun, Adv. Funct. Mater., 2016, 26, 569-576.

9 Z. J. Wang, Y. Wang and G. J. Liu, Angew. Chem., Int. Ed., 2016, 55, 1291-1294.

10 Z. J. Wang, M. Lehtinen and G. J. Liu, Angew. Chem., Int. Ed., 2017, 56, 12892-12897.

11 J. Molina, RSC Adv., 2016, 6, 68261-68291.

12 D. P. Hansora, N. G. Shimpi and S. Mishra, RSC Adv., 2015, 5, 107716-107770.

13 A. K. Yetisen, H. Qu, A. Manbachi, H. Butt, M. R. Dokmeci, J. P. Hinestroza, M. Skorobogatiy, A. Khademhosseini and S. H. Yun, ACS Nano, 2016, 10, 3042-3068.

14 J. Chen, J. Xu, K. Wang, X. Qian and R. Sun, ACS Appl. Mater. Interfaces, 2015, 7, 15641-15648.

$15 \mathrm{~J}$. Alongi and G. Malucelli, RSC Adv., 2015, 5, 24239-24263.

16 S. Y. Liang, N. M. Neisius and S. Gaan, Prog. Org. Coat., 2013, 76, 1642-1665.

17 K. A. Salmeia, S. Gaan and G. Malucelli, Polymers, 2016, 8, 319. 18 J. Alongi, F. Carosio and P. Kiekens, Polymers, 2016, 8, 357. 19 G. Malucelli, Coatings, 2016, 6, 33.

20 M. E. Mngomezulu, M. J. John, V. Jacobs and A. S. Luyt, Carbohydr. Polym., 2014, 111, 149-182.

21 S. Basak and S. W. Ali, Polym. Degrad. Stab., 2016, 133, 47-64.

22 Z. Shariatinia, N. Javeri and S. Shekarriz, Carbohydr. Polym., 2015, 118, 183-198.

23 K. Xie, A. Gao and Y. Zhang, Carbohydr. Polym., 2013, 98, 706-710.

24 D. Z. Chen, F. X. Chen, H. W. Zhang, X. Z. Yin and Y. S. Zhou, Cellulose, 2016, 23, 941-953.

25 W. W. Gao, G. X. Zhang and F. X. Zhang, Cellulose, 2015, 22, 2787-2796.

26 C. H. Xue, L. Zhang, P. Wei and S. T. Jia, Cellulose, 2016, 23, 1471-1480.

27 D. D. Zheng, J. F. Zhou, L. Zhong, F. X. Zhang and G. X. Zhang, Cellulose, 2016, 23, 2211-2220.
28 S. S. Chen, X. Li, Y. Li and J. Q. Sun, ACS Nano, 2015, 9, 40704076.

29 Y. C. Li, J. Schulz and J. C. Grunlan, ACS Appl. Mater. Interfaces, 2009, 1, 2338-2347.

30 Y. C. Li, S. Mannen, A. B. Morgan, S. Chang, Y.-H. Yang, B. Condon and J. C. Grunlan, Adv. Mater., 2011, 23, 3926-3931.

31 J. Alongi, F. Carosio, A. Frache and G. Malucelli, Carbohydr. Polym., 2013, 92, 114-119.

32 F. Carosio, C. Negrell-Guirao, A. Di-Blasio, J. Alongi, G. David and G. Camino, Carbohydr. Polym., 2015, 115, 752-759.

33 J. C. Yang, W. Liao, S. B. Deng, Z. J. Cao and Y. Z. Wang, Carbohydr. Polym., 2016, 151, 434-440.

34 F. Fang, B. Tong, T. X. Du, X. Zhang, Y. D. Meng, X. L. Liu and X. Y. Tian, Cellulose, 2016, 23, 3341-3354.

35 H. Pan, W. Wang, Y. Pan, W. Zeng, J. Zhan, L. Song, Y. Hu and K. M. Liew, Cellulose, 2015, 22, 911-923.

36 S. Chang, R. P. Slopek, B. Condon and J. C. Grunlan, Ind. Eng. Chem. Res., 2014, 53, 3805-3812.

37 K. Ariga, J. P. Hill and Q. Ji, Phys. Chem. Chem. Phys., 2007, 9, 2319-2340.

38 D. Caschera, R. G. Toro, F. Federici, C. Riccucci, G. M. Ingo, G. Gigli and B. Cortese, Cellulose, 2015, 22, 2797-2809.

39 J. Alongi, M. Ciobanu and G. Malucelli, Carbohydr. Polym., 2011, 85, 599-608.

40 J. Alongi, M. Ciobanu and G. Malucelli, Carbohydr. Polym., 2012, 87, 2093-2099.

41 J. Alongi, M. Ciobanu and G. Malucelli, Carbohydr. Polym., 2012, 87, 627-635.

42 A. El-Shafei, M. ElShemy and A. Abou-Okeil, Carbohydr. Polym., 2015, 118, 83-90.

43 S. J. Wan, L. Wang, X. J. Xu, C. H. Zhao and X. D. Liu, Soft Matter, 2014, 10, 903-910.

44 G. H. Xi, J. Wang, G. Y. Luo, Y. H. Zhu, W. C. Fan, M. Q. Huang, H. Q. Wang and X. D. Liu, Cellulose, 2016, 23, 915-927.

45 L. Wang, G. H. Xi, S. J. Wan, C. H. Zhao and X. D. Liu, Cellulose, 2014, 21, 2983-2994.

46 G. H. Xi, W. C. Fan, L. Wang, X. D. Liu and T. Endo, J. Polym. Sci., Part A: Polym. Chem., 2015, 53, 1862-1871.

47 W. C. Fan, Y. H. Zhu, G. H. Xi, M. Q. Huang and X. D. Liu, J. Appl. Polym. Sci., 2016, 133, 43024.

48 M. Lewin, J. Zhang, E. Pearce and M. Zammarano, Polym. Adv. Technol., 2010, 21, 825-834.

49 Y. Yuan, A. Siegmann and M. Narkis, J. Appl. Polym. Sci., 2010, 60, 1475-1481.

50 J. Goldshtein, T. Lublin-Tennenbaum and S. Margel, Polym. Int., 2011, 60, 1587-1593.

51 E. M. Gutman and A. L. Bobovitch, J. Therm. Anal. Calorim., 1998, 51, 245-250.

52 E. Gutman, A. Bobovitch, A. Pinski, L. Utevski, D. Sondak and M. Muskatel, J. Therm. Anal., 1996, 46, 1541-1550.

53 L. Melamed, E. Eden, M. Leifer and P. Georlette, Fire Technol., 2015, 51, 41-52.

54 G. D. Merfeld, T. T. Maa, K. Chan and D. R. Paul, Polymer, 2000, 41, 663-674.

55 A. Varesano, B. Antognozzi and C. Tonin, Synth. Met., 2010, 160, 1683-1687. 